\title{
Early stage breast cancer with concomittant primary hyperparathyroidism and auto- immune thrombocytopenia: a case report
}

\author{
BP Phakathi, A Mannell, S Nietz
}

\begin{abstract}
Summary: Co-existence of early stage breast cancer, primary hyperparathyroidism and autoimmune thrombocytopenia has never been reported. While there is an increased prevalence of primary hyperparathyroidism in young patients with breast cancer, this is the first case report of early breast cancer with both primary hyperthyroidism and autoimmune thrombocytopenia.
\end{abstract}

S Afr J Surg 2018;56(2)

http://dx.doi.org/10.17159/2078-5151/2018/v56n1a2191

\section{Case report}

A 42year-old female presented to Charlotte Maxeke Johannesburg Academic Hospital Breast Clinic with a monthlong history of a painless lump in her right breast. Stage IIA (T2N0M0), luminal A right breast cancer was diagnosed. As part of the metastatic work-up, the following abnormalities were detected: a high corrected serum calcium: $2.87 \mathrm{mmol} / 1$ (Normal range: $2-2.5 \mathrm{mmol} / \mathrm{l}$ ), a decrease level of inorganic phosphate of $0.4 \mathrm{mmol} / \mathrm{l}(0.78-1.42 \mathrm{mmol} / \mathrm{l})$ with a normal magnesium level. Full blood count revealed an isolated thrombocytopenia with the platelet count of $39 \times 10^{9} / 1$ (Normal range: $186-454 \times 10^{9}$ ). The differential count of blood cells showed decrease in platelet numbers with no obvious platelet clumping. Renal function tests showed a mild renal impairment (Urea: $7.1 \mathrm{mmol} / \mathrm{l}$; Creatinine $113 \mathrm{umol} / \mathrm{l}$ and GFR: 46ml/min/1.73m²). There were no symptoms or signs to suggest bone metastases or a bleeding disorder. There was no history of heparin administration at the time thrombocytopenia was diagnosed. The patient was HIV negative with no previous drug history and had not had any recent infection. Viral serology (CMV and EBV) was also negative. The abdominal ultrasound showed no evidence of liver metastases but revealed a right hydronephrosis. A CTIntravenous pyelogram scan demonstrated right renal and ureteric stones. A 99mTechnitium bone scan was done and excluded osteolytic metastases. Parathyroid hormone (PTH) was done and was elevated at 84 pmol/l. A Sestamibi scan identified a left inferior parathyroid adenoma.

To investigate the cause of thrombocytopenia, the patient underwent a bone marrow trephine and aspiration biopsy. This showed a normocellular marrow with no evidence of metastatic breast carcinoma or other diseases which could result in persistent thrombocytopenia. There was no evidence of splenomegaly on abdominal ultrasound.

Platelets were transfused prior to a sentinel lymph node biopsy of the right axilla. A double $\mathrm{J}$ stent was placed to relieve the right ureteric obstruction. The patient underwent a focused left inferior parathyroidectomy and right mastectomy with right axillary lymph node dissection. Histopathological confirmed a parathyroid adenoma and a completely excised invasive breast carcinoma with lymph node metastases (pT2pN1M0).

Transient postoperative hypocalcaemia required intravenous and oral calcium supplements. On discharge, the corrected serum calcium was normal $(2.23 \mathrm{mmol} / \mathrm{l})$, the platelet count had stabilised at $251 \times 10^{9} / 1$ and the parathyroid hormone level was $8.7 \mathrm{pmol} / \mathrm{l}$. The patient did not require steroid therapy for the thrombocytopenia.

\section{Discussion}

Hypercalcaemia occurs in about $30-40 \%$ of cases of patients with breast cancer and it is usually due to osteolytic metastases as well as the activity of a circulating parathyroid hormone (PTH)-related peptide secreted by a tumour. ${ }^{1}$ There is a relationship between breast cancer and primary hyperparathyroidism though the mechanism remains unclear. ${ }^{2}$ Likewise, an auto-immune thrombocytopenia, in the absence of bone marrow infiltration, cytotoxic suppression, drugs, and viral infections, has been described in solid tumours including breast cancer. ${ }^{3}$ Concurrence of these three pathologies has not been described.

An increased prevalence of parathyroid adenoma has been noted in women after treatment for early breast cancer. ${ }^{1}$ Fierabracci et al. identified 7 women with primary hyperparathyroidism among a hundred patients with breast cancer. Six of these patients underwent a neck exploration to remove a parathyroid adenoma. ${ }^{1}$ Following multiple reports of primary hyperparathyroidism in the absence of metastatic disease, this association was further explored in a Swedish study of 9835 women after excision of parathyroid 
adenomas. ${ }^{2}$ A greater than expected incidence of breast cancer was found on follow-up of this population group, the increased risk of breast cancer persisting for more than a decade after the parathyroid surgery.

The possible existence of a genetic predisposition to both breast cancer and primary hyperparathyroidism was shown in a study of 675 female patients with Multiple Endocrine Neoplasia Type 1 (MEN1); the condition is characterised by parathyroid, pituitary and pancreatic islet cell tumours.$^{3}$ The international Breast Cancer in MEN1 Study Group has shown these women to have an increased risk of breast cancer. The germ line mutation in MEN1 tumour suppressor gene, which encodes for menin, a co-regulator of estrogen receptor-a, may underlie this association. ${ }^{3}$

The possibility of an underlying MEN 1 syndrome was not able to be investigated in this patient as she defaulted from follow-up after surgery. However, this case highlights the importance of PTH estimation where hypercalcaemia is found in early breast cancer.

Thrombocytopenia is an uncommon complication of solid tumours including breast cancer. ${ }^{4}$ In these cases, it is usually due to metastatic replacement of bone marrow, the toxic effects of chemotherapy on the marrow or platelet consumption by tumoural factors activating disseminated intravascular coagulation, paraneoplastic syndrome including autoimmune thrombocytopenia and idiopathic thrombocytopenic purpura. ${ }^{3,4}$

Auto-immune thrombocytopenia is an uncommon cause of thrombocytopenia in solid tumours and is a diagnosis by exclusion of the other causes. ${ }^{4}$ It is most frequently associated with lung and breast cancers. ${ }^{3}$ In about $50 \%$ of cases, it coexists with the cancer while in other documented cases, it has preceded the diagnosis of malignancy or has been a late presentation after the treatment of the tumour. ${ }^{4}$ In the latter, auto-immune thrombocytopenia is associated with cancer recurrence. ${ }^{4}$

Thrombocytopenia may resolve following a resection of the cancer but steroid therapy may be needed..$^{5}$ There are reports of simultaneous occurrence of immune thrombocytopenia and breast cancer where the platelet count was improved or stabilised by either anticancer therapy or the commonly used treatments for immune thrombocytopenia. ${ }^{5}$ In this patient a paraneoplastic autoimmune thrombocytopenia is a possible explanation as excision of the breast cancer resulted in the restoration and stabilisation of a normal platelet count in the short term.

\section{Conclusion}

Primary hyperparathyroidism and an auto-immune thrombocytopenia are rarely associated with breast cancer. In this report, the unique occurrence of these three conditions is described. In non-metastatic breast cancer, hypercalcaemia requires parathyroid hormone estimation. In isolated thrombocytopenia when other causes have been excluded, a paraneoplastic auto-immune thrombocytopenia may be considered. In early stage breast cancer, with primary hyperparathyroidism, autoimmune thrombocytopenia is not a contra-indication to surgical treatment.

\section{REFERENCES}

1. Fierabracci P, Pinchera A, Miccoli P et al. Increased prevalence of primary hyperparathyroidism in treated breast cancer. J Endocrinol Invest. 2001;24:315-20.

2. Rao V, Chaukar D, D'Cruz A. Hypercalcemia and treated breast cancer: The Diagnostic dilemma. J Cancer Res Ther. 2009;5(1):46-8.

3. Dreijerink KM, Goudet P, Burgess JR, et al. Breast Cancer predisposition with Multiple Endocrine Neoplasia Type 1. N Engl J Med. 2014;371:6 (Letter to the editor).

4. Krauth M, Puthenparambil J, Lechner K. Paraneoplastic autoimmune thrombocytopenia in solid tumours. Crit Review Oncology/Hematology. 2012;81:75-81.

5. Samimi AM, Mirkheshti N, Heidarpour M, et al. Idiopathic thrombocytopenic purpura in women with breast cancer. J Res Med Sci. 2010;15(6):359-63. 